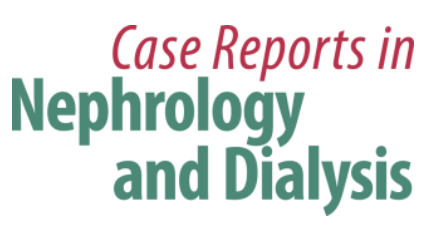

Case Rep Nephrol Dial 2019;9:102-107

DOI: 10.1159/000502336

Published online: August 20, 2019

(C) 2019 The Author(s)

Published by S. Karger AG, Base

www.karger.com/cnd

This article is licensed under the Creative Commons Attribution-NonCommercial 4.0 International License (CC BY-NC) (http://www.karger.com/Services/OpenAccessLicense). Usage and distribution for commercial purposes requires written permission.

\title{
Faecal Microbiota Transplantation Eradicated Extended-Spectrum Beta-Lactamase-Producing Klebsiella pneumoniae from a Renal Transplant Recipient with Recurrent Urinary Tract Infections
}

\author{
Anne Karmisholt Grosen ${ }^{a} \quad$ Johan Vestergaard Povlsen ${ }^{b}$ \\ Lars Erik Lemming ${ }^{a} \quad$ Simon Mark Dahl Jørgensen ${ }^{c}$ \\ Jens Frederik Dahlerup ${ }^{c}$ Christian Lodberg Hvas ${ }^{c}$ \\ aDepartment of Clinical Microbiology, Aarhus University Hospital, Aarhus, Denmark; \\ bDepartment of Renal Medicine, Aarhus University Hospital, Aarhus, Denmark; \\ 'Department of Hepatology and Gastroenterology, Aarhus University Hospital, \\ Aarhus, Denmark
}

\section{Keywords}

Renal transplantation - Extended-spectrum beta-lactamase-producing Klebsiella pneumoniae - Multidrug-resistant microorganisms - Faecal microbiota transplantation · Intestinal decolonisation

\begin{abstract}
Renal transplant recipients (RTRs) are highly susceptible to infections, and antimicrobial resistance is an increasing problem with limited treatment options. Faecal microbiota transplantation (FMT) is effective for recurrent Clostridium difficile infection and may be used for patients with intestinal carriage of multidrug-resistant (MDR) microorganisms. We present a RTR who suffered from recurrent urinary tract infections (UTIs) caused by extended-spectrum beta-lactamase-producing $(\mathrm{ESBL}+)$ Klebsiella pneumoniae. Blood and urinary isolates revealed the
\end{abstract}




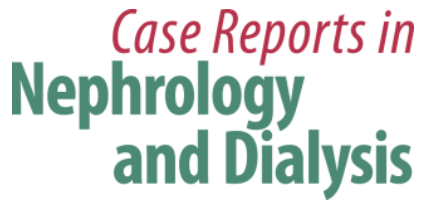

Case Rep Nephrol Dial 2019;9:102-107

DOI: $10.1159 / 000502336$

(c) 2019 The Author(s). Published by S. Karger AG, Basel www.karger.com/cnd

Grosen et al.: Faecal Microbiota Transplantation for Extended-Spectrum Beta-Lactamase

same antibiotic susceptibility pattern, and whole-genome sequencing confirmed identical isolates in blood and urine. Despite several treatments with meropenem, the patient experienced recurrent infections that caused hospitalisation. ESBL+ K. pneumoniae was isolated in faeces. In an attempt to decolonise the gut, FMT was performed. A few days after nasojejunal infusion of donor faeces, the patient experienced a single relapse of UTI. During the subsequent 12 months, no further episodes of UTI occurred. Absence of ESBL+ K. pneumoniae in urine and faeces was demonstrated during follow-up. We conclude that FMT may be an effective treatment in RTRs with recurrent UTIs caused by intestinal colonisation with MDR organisms.

C 2019 The Author(s)
Published by S. Karger AG, Basel

\section{Introduction}

Renal transplant recipients (RTRs) are highly susceptible to infections due to immunosuppressive therapy. Common risk factors are diabetes mellitus, high age, use of catheters, and extended hospitalisation $[1,2]$. Infectious complications following renal transplantation (RTX) may worsen graft function and are associated with prolonged hospitalisation and increased medication costs, morbidity, and mortality [1,3]. Urinary tract infection (UTI) is the most common bacterial infection after RTX [1-5]. Urinary isolates from RTRs have revealed a significant increase in antibiotic resistance rates, particularly with Klebsiella pneumoniae [4]. The burden of UTIs caused by K. pneumoniae that produce extended-spectrum beta-lactamase (ESBL+) after RTX is an emerging problem [4-6]. Compatible with the possibility of conversion of intestinal carriage into active UTI, a strong association between gut ESBL+ K. pneumoniae colonisation and ESBL+ K. pneumoniae UTIs in RTRs has been demonstrated [5].

Faecal microbiota transplantation (FMT) is a highly effective and established treatment for recurrent Clostridium difficile infection [7, 8]. FMT is being considered for other indications where a disrupted intestinal microbiota may contribute to disease pathogenesis [7], and it is an emerging treatment modality for patients with intestinal carriage of multidrug-resistant (MDR) microorganisms [9-12]. Antimicrobial resistance is an imminent challenge to global public health, and the problem is increasing. The treatment options for MDR bacterial infections are limited [13].

\section{Case Report}

A 64-year-old Caucasian man with chronic kidney disease secondary to diabetic nephropathy underwent cadaveric renal allotransplantation in 2017. He received standard antibacterial prophylaxis with perioperative cefuroxime and a 6-month treatment with trimethoprimsulfamethoxazole. The immunosuppressive treatment consisted of tacrolimus $(0.1 \mathrm{mg} / \mathrm{kg}$ twice daily), mycophenolate mofetil (750 mg twice daily), prednisolone (tapered from $20 \mathrm{mg}$ once daily), and basiliximab ( $20 \mathrm{mg}$ ) just before RTX and on day 4 after RTX as induction therapy. Due to recurrent infections, immunosuppression was gradually reduced. Prednisolone was stopped before FMT. Mycophenolate mofetil was reduced to $500 \mathrm{mg}$ twice daily 51 days after RTX and further to $250 \mathrm{mg}$ twice daily 81 days after RTX. Tacrolimus was gradually reduced to $1 \mathrm{mg}$ twice daily, corresponding to a trough level of approximately $5 \mu \mathrm{g} / \mathrm{L}$. A ureteral stent was inserted at RTX and removed 1 month later. The patient never achieved good graft function (Fig. 1). Graft biopsies made due to rising levels of serum creatinine showed interstitial fibrosis, tubular atrophy, and increasing numbers of sclerotic glomeruli, but no histo- 


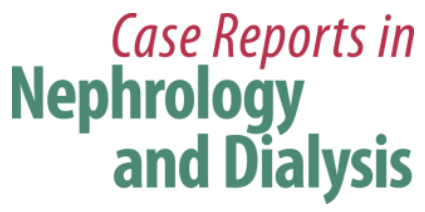

logical signs of cellular or humoral rejection 2 and 3 months after RTX. Donor-specific antibodies were never detected.

Eighty days after RTX the patient was admitted with urosepsis. ESBL+ K. pneumoniae was isolated in blood and urine. During the subsequent 5 months, he was hospitalised seven times because of UTI (Fig. 1). Each episode of UTI was associated with a further decline in graft function (Fig. 1). At each admission, ESBL+ K. pneumoniae only fully susceptible to meropenem and ceftazidime-avibactam was isolated in urinary samples. Antimicrobial susceptibility was interpreted according to the European Committee for Antimicrobial Susceptibility Testing. ESBL+ K. pneumoniae bacteraemia occurred during two of the admissions. Blood and urinary isolates showed the same antibiotic susceptibility pattern, and whole-genome sequencing confirmed identical isolates in blood and urine.

Standard empiric therapy with piperacillin-tazobactam was initiated at the first three admissions. Based on urinary and blood culture results, antibiotic treatment was changed to meropenem with a median treatment duration of 12 days (range 4-16). Despite a rapid treatment response, UTI recurrence occurred following withdrawal of the antibiotic treatment, with a median of 7 days (range 4-18) from withdrawal to re-admission (Fig. 1).

A positron emission tomography-computed tomography scan performed 6 months after RTX showed no focal uptake pattern or urinary tract foci. It was hypothesised that gastrointestinal colonisation with ESBL+ K. pneumoniae was the cause of reinfection. A faecal sample made 7 months after RTX confirmed ESBL+ K. pneumoniae carriage.

Eight months after RTX, the patient received FMT in an attempt to decolonise the ESBL+ K. pneumoniae from the gut. Prior to FMT, the patient further tested positive for $C$. difficile toxin $\mathrm{A}$ and $\mathrm{B}$, and consequently treatment with vancomycin $125 \mathrm{mg}$ orally four times per day was initiated 3 days prior to FMT. After FMT, resolution of diarrhoea was achieved. FMT was administered by a nasojejunal tube 2 weeks after the last course of meropenem. No adverse events related to FMT were observed.

FMT was performed within the local clinical FMT framework in compliance with the Danish Tissue Act [7]. Donor faeces were obtained from a healthy blood donor after approved screening results and an approved medical consultation. The screening of the faeces donor consisted of a questionnaire, blood sampling, and faecal sample analysis. A medical consultation was done to verify the consistency between the approved screening results and the overall state of health. At the laboratory, the donated material was cryopreserved, and thawing procedures were performed before the clinical use of donor faeces.

Six days after FMT, the patient was re-admitted with UTI relapse due to ESBL+ K. pneumoniae identical with that formerly isolated from urine and blood and successfully treated with meropenem. Subsequently, no further episodes of UTI occurred (Fig. 1). The patient was followed during the first 12 months after FMT, and he was without any symptoms of UTI. No further admissions occurred. ESBL+ K. pneumoniae could not be isolated in urinary or faecal samples collected at outpatient follow-up 4 and 8 months after FMT.

\section{Discussion}

This case report provides proof of concept that intestinal carriage of MDR microorganisms may be eradicated using FMT. In this particular patient, we believe that FMT prevented further episodes of UTI and C. difficile infection. Reduction of immunosuppression may have facilitated the clinical effect of FMT. 


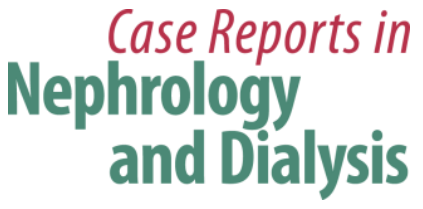

Case Rep Nephrol Dial 2019;9:102-107

DOI: $10.1159 / 000502336$

(c) 2019 The Author(s). Published by S. Karger AG, Basel www.karger.com/cnd

Grosen et al.: Faecal Microbiota Transplantation for Extended-Spectrum Beta-Lactamase

The rational basis for believing that FMT caused the eradication of ESBL+ K. pneumoniae is that colonic carrier status caused the recurrent infections and that colonisation was cleared following FMT. One plausible explanation of the single relapse of UTI shortly after FMT might be that restoration of the disrupted intestinal microbiota takes time. The presented RTR had several factors that contributed to UTI risk, including immunosuppressive treatment, diabetes mellitus, and placement of a urinary catheter. Rectal carriage of ESBL+ K. pneumoniae is associated with UTIs in RTRs [5], supporting the assumption that intestinal colonisation with ESBL+ K. pneumoniae is a predisposing factor for UTIs in RTRs.

A shift in the faecal microbiota composition towards the donor microbial composition as well as restoration of microbial diversity after FMT has previously been documented [12]. In a recent study, 15 patients carrying ESBL+ Enterobacteriaceae were treated with FMT in an attempt to achieve intestinal decolonisation, and $20 \%$ of the patients became ESBL-negative after the first FMT procedure. Seven patients underwent a second FMT resulting in a intestinal decolonisation rate of $40 \%$, suggesting that repeated FMT procedures increase treatment effectivity [12]. In the present case, a single FMT procedure was sufficient.

RTRs constitute an immunosuppressed population, and the increase in antibacterial resistance is an alarming trend in this population. The incidence of ESBL+ Enterobacteriaceae in solid organ transplant recipients has been documented to increase from 13 to 38 to $45 \%$ for the first, second, and third UTI episodes, respectively [10]. The intestine is the primary reservoir for colonisation with MDR Enterobacteriaceae. Based on this, there is an urgent clinical need for an approach to achieve intestinal decolonisation, particularly among vulnerable patients, including RTRs.

Eradication of intestinal ESBL+ and carbapenemase-producing Enterobacteriaceae carriage using FMT was tested in a recent randomised multicentre clinical trial in immunocompetent patients [14]. Of 64 planned patients, 39 were randomised to a 5-day course of oral antibiotics followed by FMT or no intervention. FMT was successful in 9/22 (41\%) versus $5 / 17$ (29\%) following no intervention, with an odds ratio of 1.7 (95\% confidence interval 0.46.4) in favour of FMT. The difference was not statistically significant, and the study was underpowered because only 39 of 64 planned patients were included. Further, immunosuppressed patients may have an increased risk of developing sustained colonisation and thereby suffer from infections.

In RTRs, the present and previously published cases support that FMT is a safe and effective treatment option to eradicate intestinal carriage of MDR microorganisms $[9,11,12]$, with subsequent reduction in antibiotics use and hospital admissions. Overall, antimicrobial exposure is associated with antimicrobial resistance [13]. A reduction in carbapenem use is an important antibiotic stewardship intervention. FMT is a preferable antimicrobial-free treatment option that may effectively reduce broad-spectrum antibiotic use and thereby hospital-acquired MDR infections as well as superinfections such as $C$. difficile infection. The potential benefits of applying FMT in patients such as the one in the present report therefore apply to both the patients in question, to antibiotic stewardship, and to health economics.

\section{Statement of Ethics}

The present study adhered to the Declaration of Helsinki. The patient gave written informed consent to the publication of this case report. The treatment was approved by the head of department. Since treatment was carried out as a part of the integrated clinical patient care, no ethics committee approval was required according to Danish law. 


\section{Case Reports in Nephrology and Dialysis}

Grosen et al.: Faecal Microbiota Transplantation for Extended-Spectrum Beta-Lactamase

\section{Disclosure Statement}

The authors declare no conflicts of interest.

\section{Funding Sources}

The study was supported by the Danish Regions Medical Fund (j.no. 14/217) and the Innovation Fund Denmark (j.no. 8056-00006B).

\section{Author Contributions}

A.K. Grosen collected microbiology data and wrote the first manuscript draft. J.V. Povlsen managed the kidney-related clinical treatment of the patient. L.E. Lemming was in charge of clinical microbiology services. S.M.D. Jørgensen participated in data analysis and presentation. J.F. Dahlerup performed all FMT-related treatments. C.L. Hvas contributed to and was overall responsible for the FMT-related treatment. All authors took part in discussion of the patient case and critically revised the manuscript. All authors approved the final version of the manuscript.

\section{References}

1 Adamska Z, Karczewski M, Cichańska L, Więckowska B, Małkiewicz T, Mahadea D, et al. Bacterial Infections in Renal Transplant Recipients. Transplant Proc. 2015 Jul-Aug;47(6):1808-12.

2 Galindo Sacristán P, Pérez Marfil A, Osorio Moratalla JM, de Gracia Guindo C, Ruiz Fuentes C, Castilla Barbosa YA, et al. Predictive factors of infection in the first year after kidney transplantation. Transplant Proc. 2013; 45(10):3620-3.

3 Hemmersbach-Miller M, Alexander BD, Sudan DL, Pieper C, Schmader KE. Single-center analysis of infectious complications in older adults during the first year after kidney transplantation. Eur J Clin Microbiol Infect Dis. 2019 Jan;38(1):141-8.

4 Origüen J, Fernández-Ruiz M, López-Medrano F, Ruiz-Merlo T, González E, Morales JM, et al. Progressive increase of resistance in Enterobacteriaceae urinary isolates from kidney transplant recipients over the past decade: narrowing of the therapeutic options. Transpl Infect Dis. 2016 Aug;18(4):575-84.

5 Wilkowski P, Gajko K, Marczak M, Hryniewiecka E, Wojtowicz M, Dobrzaniecka K, et al. Clinical Significance of Gastrointestinal Carriage of Klebsiella Pneumoniae-Producing Extended-Spectrum Beta-Lactamases in Kidney Graft Recipients. Transplant Proc. 2018 Jul-Aug;50(6):1874-7.

6 Linares L, Cervera C, Hoyo I, Sanclemente G, Marco F, Cofán F, et al. Klebsiella pneumoniae infection in solid organ transplant recipients: epidemiology and antibiotic resistance. Transplant Proc. 2010 Oct;42(8):29413.

7 Jørgensen SMD, Hansen MM, Erikstrup C, Dahlerup JF, Hvas CL. Faecal microbiota transplantation: establishment of a clinical application framework. Eur J Gastroenterol Hepatol. 2017 Nov;29(11):e36-45.

8 van Nood E, Vrieze A, Nieuwdorp M, Fuentes S, Zoetendal EG, de Vos WM, et al. Duodenal infusion of donor feces for recurrent Clostridium difficile. N Engl J Med. 2013 Jan;368(5):407-15.

9 Singh R, van Nood E, Nieuwdorp M, van Dam B, ten Berge IJ, Geerlings SE, et al. Donor feces infusion for eradication of Extended Spectrum beta-Lactamase producing Escherichia coli in a patient with end stage renal disease. Clin Microbiol Infect. 2014 Nov;20(11):0977-8.

10 Manges AR, Steiner TS, Wright AJ. Fecal microbiota transplantation for the intestinal decolonization of extensively antimicrobial-resistant opportunistic pathogens: a review. Infect Dis (Lond). 2016 Aug;48(8): 587-92.

11 Stalenhoef JE, Terveer EM, Knetsch CW, Van't Hof PJ, Vlasveld IN, Keller JJ, et al. Fecal Microbiota Transfer for Multidrug-Resistant Gram-Negatives: A Clinical Success Combined With Microbiological Failure. Open Forum Infect Dis. 2017 Mar;4(2):ofx047. 


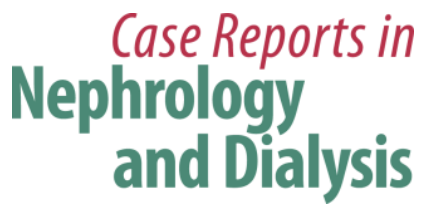
Case Rep Nephrol Dial 2019;9:102-107
DOI: $10.1159 / 000502336$
(c) 2019 The Author(s). Published by S. Karger AG, Basel www.karger.com/cnd

Grosen et al.: Faecal Microbiota Transplantation for Extended-Spectrum Beta-Lactamase

12 Singh R, de Groot PF, Geerlings SE, Hodiamont CJ, Belzer C, Berge IJ, et al. Fecal microbiota transplantation against intestinal colonization by extended spectrum beta-lactamase producing Enterobacteriaceae: a proof of principle study. BMC Res Notes. 2018 Mar;11(1):190.

13 World Health Organization. Worldwide Country Situation Analysis: Response to Antimicrobial Resistance. Geneva: World Health Organization; 2015. www.who.int/drugresistance/documents/situationanalysis/en/.

14 Huttner BD, de Lastours V, Wassenberg M, Maharshak N, Mauris A, Galperine T, et al. A 5-day course of oral antibiotics followed by faecal transplantation to eradicate carriage of multidrug-resistant Enterobacteriaceae: a randomized clinical trial. Clin Microbiol Infect. 2019 Jul;25(7):830-8.

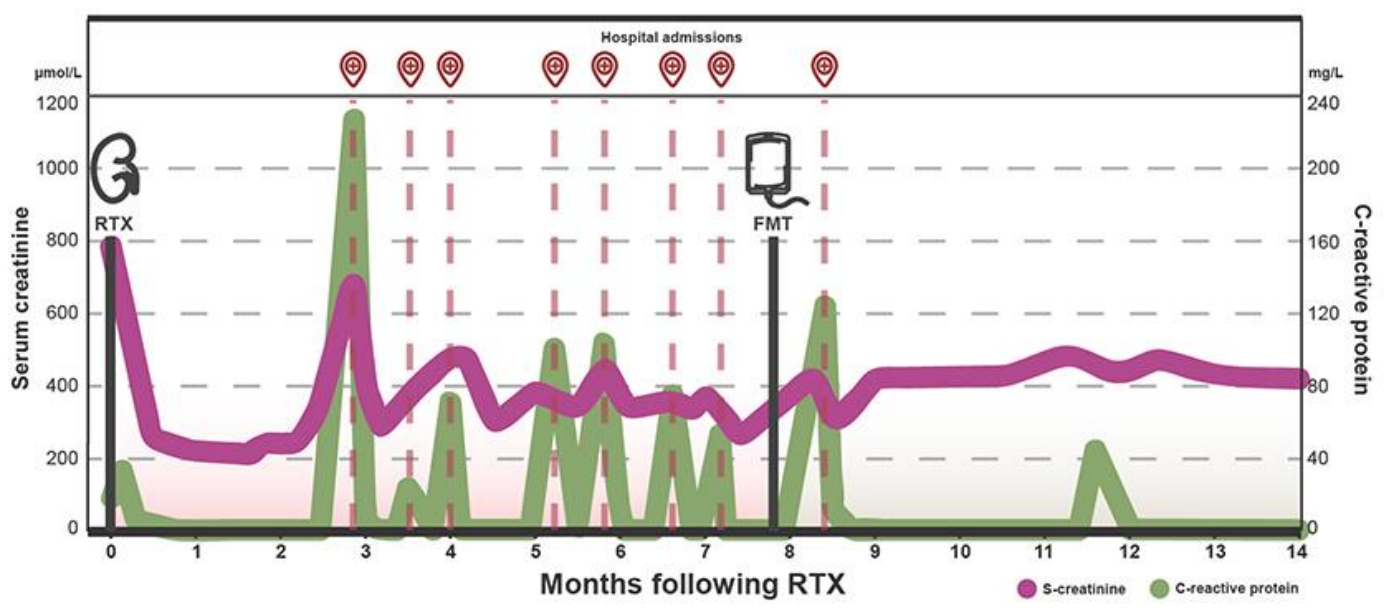

Fig. 1. Time line of C-reactive protein and serum creatinine in relation to RTX and FMT. Hospital admissions due to urinary tract infections caused by extended-spectrum beta-lactamase-producing K. pneumoniae are visualised. FMT, faecal microbiota transplantation; RTX, renal transplantation. 